\title{
ALGORITMOS GENÉTICOS E DE EVOLUÇÃO DIFERENCIAL APLICADOS À OTIMIZAÇÃO DE CONTROLADORES UTILIZANDO A NORMA $H_{2}$ COMO CRITÉRIO DE DESEMPENHO
}

\author{
ANA BeAtriz Rodrigues ${ }^{1}$, Cristiano Marcos AgUlHari ${ }^{1}$ \\ 1. Laboratório de Controle e Otimização de Sistemas, Departamento de Engenharia Elétrica, \\ Universidade Tecnológica Federal do Paraná \\ Av. Alberto Carazai, 1640 - Vila Seugling, Cornélio Procópio - PR, 86300-000 \\ E-mails: anarealunos.utfpr.edu.br, agulharieutfpr.edu.br
}

\begin{abstract}
The controller synthesis using the $H_{2}$ norm as performance criterion is usually solved either using conditions based on linear matrix inequalities or through the resolution of Riccati equations. However, conservative results may arise if other constraints, or even if non-convex design criteria, are incorporated. In this sense, this work proposes to analyze the efficiency of heuristics by the application of evolutionary algorithms in the synthesis of controllers using $H_{2}$ norm as a performance criterion. The controllers obtained by genetic and differential evolution algorithms are simulated computationally by MATLAB / Simulink ${ }^{\circledR}$ software, and also tested experimentally into a torsional system. It is also necessary to evaluate the closed-loop physical implementations of this system and to analyze if it is necessary to add different constraints to assure the implementability of the obtained controller. It is important to emphasize that the implemented algorithms can be easily adapted to any system, if the mathematical model is known.
\end{abstract}

Keywords— Genetic Algorithm. Differential Evolution Algorithm. $H_{2}$ Norm. MATLAB/Simulink. Torsional system.

Resumo - A síntese de controladores utilizando a norma $\mathrm{H}_{2}$ como critério de desempenho é comumente solucionada a partir de condições baseadas em desigualdades matriciais lineares ou por meio da resolução de equações de Riccati. No entanto, tais abordagens podem trazer resultados conservadores caso outras restrições ou critérios de projeto não-convexos sejam incorporadas. Neste sentido, este trabalho propõe analisar a eficiência de heurísticas pela aplicação de algoritmos evolutivos na síntese de controles utilizando a norma $\mathrm{H}_{2}$ como critério de desempenho, a fim de simplificar suas soluções. Os controladores obtidos pelos algoritmos genéticos e de evolução diferencial são simulados computacionalmente pelo software MATLAB/Simulink®, e também testados experimentalmente em um sistema torcional. Necessita-se também avaliar as implementações físicas em malha fechada deste sistema e analisar se há a necessidade de acrescentar diferentes restrições para garantir a implementabilidade do controlador obtido. É importante ressaltar que os algoritmos implementados podem ser facilmente adaptados para qualquer sistema, se seu modelo matemático for conhecido.

Palavras-chave— Algoritmo genético. Algoritmo de evolução diferencial. Norma $H_{2}$. MATLAB/Simulink. Sistema torcional.

\section{Introdução}

Em processos industriais é comum o uso de plantas e sistemas de controles projetados a fim de atingir mais de uma especificação de trabalho, como por exemplo, um sistema que necessite de robustez a ruídos otimizando certos critérios de desempenho. Uma das formas de atingir tais objetivos é modelar o desempenho pela minimização de sua norma $H_{2}$ enquanto que a robustez necessária pode ser garantida por meio de sua norma $H_{\infty}$ (CHUN; CHOI; KIM, 2017).

A abordagem mais utilizada para a síntese de controladores mistos $H_{2} / H_{\infty}$ é baseada na resolução de um problema de otimização baseado em desigualdades matriciais lineares (Linear Matrix Inequalities (LMI)) (BOYD et al., 1994). O maior problema desta abordagem é que as condições menos conservadoras normalmente dependem de vários parâmetros que devem ser pré-determinados. Outro problema apresentado a este tipo de resolução é que certas restrições de projeto podem ser não-convexas, o que aumenta veemente a complexidade desta abordagem (WU et al., 2013).

O algoritmo apresentado por Gonçalves, Palhares e Takahashi (2004) possibilita uma eficiente síntese do controle misto robusto a partir de realimentação de estados, por meio da resolução de um conjunto de LMIs. Outros trabalhos desenvolveram pesquisas sobre a resolução de LMIs para a obtenção da síntese de controladores mistos como apresentado em Boyd et al. (1994), Ghaoui e Niculescu (2000), Leibfritz (2001), Yu (2004), Sato (2010) e também em Andrade e Agulhari (2016). Nota-se que há diversas pesquisas a respeito, mas a grande maioria é centrada na resolução de LMIs, o que torna interessante pesquisar e analisar outros tipos de abordagens.

Por outro lado, há diversas aplicações industriais onde o sistema necessita de alto desempenho e sua robustez a perturbações já é tratada por outros agentes, portanto a síntese do controlador não precisa considerar este critério. Para estas aplicações, é possível analisar e otimizar apenas o critério de desempenho do sistema de controle associado a norma $\mathrm{H}_{2}$, tornando sua resolução mais simples e eficiente. A solução mais comum para a otimização da norma $\mathrm{H}_{2}$ associado ao desempenho de sistemas de controle é a partir das equações algébricas de Riccati. Em Boers, Weiland e Damen (1997) é apresentado um estudo comparativo entre a solução de Riccati e uma distribuição probabilística proposta pelos autores.

As equações de Riccati podem também ser modeladas em termos de LMIs, que, por sua vez, é uma das abordagens mais utilizadas para resolver o problema 
de minimização da norma $H_{2}$. Alguns trabalhos propuseram tal resolução para a otimização de sistemas de controle como os apresentados em Farias et al. (2000), Xie e Chen (2010) e Gomez et al. (2015).

Neste contexto, a principal contribuição deste trabalho é a aplicação do algoritmo genético (AG) e do algoritmo de evolução diferencial (ED) na otimização do critério de desempenho relacionado à norma $\mathrm{H}_{2}$ de controladores, uma vez que os autores não encontraram em literatura a aplicação do ED neste tipo de modulação de problema de controle. A comparação com o AG se faz conveniente por ser uma ferramenta heurística clássica, porém pouco utilizada na aplicação descrita, podendo assim, comprovar a eficiência de ambos algoritmos.

\section{Norma $\mathrm{H}_{2}$}

Projetos de sistemas de controle são realizados a fim de que o sistema estudado apresente determinadas especificações de funcionamento e desempenho. Assim, uma das maneiras mais utilizadas para classificar o desempenho de um sistema é uma medida de energia para determinados sinais de interesse (TROFINO; COUTINHO; BARBOSA, 2005).

A norma $\mathrm{H}_{2}$, definida formalmente na sequência, é uma medida da energia média do sistema. Para que a norma $\mathrm{H}_{2}$ seja utilizada como um índice de desempenho ou custo funcional do sistema em malha fechada, é introduzida uma saída artificial a ser controlada neste sistema. A resolução deste sistema em malha fechada é equivalente à substituição de suas condições iniciais por uma perturbação persistente nãocorrelacionada entrando em todas as direções do espaço de estados (TROFINO; COUTINHO; BARBOSA, 2005). Considere o sistema descrito por (1), também denominado de planta generalizada.

$$
G(t)=\left\{\begin{array}{l}
\dot{x}(t)=A x(t)+B u(t)+B_{w} w(t) \\
y(t)=C x(t) \\
z(t)=C_{z} x(t)+D_{z} u(t)+D_{z w} w(t)
\end{array}\right\}
$$

As variáveis na Equação são: $x(t)$ as variáveis de estado; $u(t)$ as variáveis de entrada; $y(t)$ as variáveis de saída; $w(t)$ a entrada exógena; $z(t)$ a saída artificial (controlada) do sistema; $A$ a matriz de estados; $B$ a matriz de entrada; $C$ a matriz de saída; $B_{w}$ a matriz relacionada ao valor resultante da norma $\mathrm{H}_{2} ; \mathrm{C}_{z}$ a matriz relacionada com a velocidade de resposta dos estados do sistema; $D_{z}$ a matriz relacionada com a energia de controle aplicada à entrada do sistema; $D_{z w}$ a matriz de transmissão direta entre a entrada exógena e a saída controlada.

A norma $\mathrm{H}_{2}$ do sistema, apresentado na Definição 1 , é uma medida comumente utilizada da energia média da saída controlada resultante da entrada.
Definição 1 (Norma $H_{2}$ ) A norma $H_{2}$ de um sistema $G(s)$ é definida por (2).

$$
\|G(s)\|_{2}^{2}=\sqrt{\frac{1}{2 \pi} \int_{-\infty}^{\infty} \operatorname{traço}\left(G(j \omega)^{T} G(j \omega)\right) d \omega}
$$

Sendo $G(j \omega)$ a resposta em frequência da função de transferência $G(s)$.

Propriedade (Norma $\mathrm{H}_{2}$ finita) A norma $\mathrm{H}_{2}$ de um sistema é finita se, e somente se, o sistema $G$ for estável e estritamente próprio, ou seja, se a matriz de estados $A$ for Hurwitz e se $D_{z w}=0$.

A norma $\mathrm{H}_{2}$ do sistema também pode ser definida conforme (3) (TROFINO; COUTINHO; BARBOSA, 2005).

$$
\|G(s)\|_{2}^{2}=\int_{-\infty}^{\infty} z(t)^{T} z(t) d t
$$

Substituindo $z(t)$ em (3) e considerando que o sistema seja Hurwitz e que $C_{z} D_{z}=0$, a Equação apresentada em (4) é similar à integral do desempenho obtida pelo Regulador Quadrático Linear (Linear Quadratic Regulator (LQR)).

$$
\|G(s)\|_{2}^{2}=\int_{-\infty}^{\infty}\left\{x^{T}(t) C_{z}^{T} C_{z} x(t)+u^{T}(t) D_{z}^{T} D_{z} u(t)\right\} d t
$$

Assim, minimizar a norma $H_{2}$ é equivalente a minimizar uma ponderação entre a energia dos estados e a energia do sinal de controle, sendo tais ponderações relacionadas, respectivamente, às matrizes $C_{z} \mathrm{e}$ $D_{z}$ (ZHOU; DOYLE; GLOVER, 1996). As matrizes $C_{z}$ e $D_{z}$, e consequentemente a variável artificial $z(t)$ , são definidas pelo usuário para determinar a ponderação de minimização dos aspectos mencionados.

Além das matrizes do sistema aumentado $C_{z} \mathrm{e}$ $D_{z}$, necessita-se determinar uma lei de controle $u(t)$ para que a norma $\mathrm{H}_{2}$ do sistema controlado seja minimizada. Para atingir este objetivo, os parâmetros do controlador que compõem $u(t)$ são determinados pelos algoritmos heurísticos propostos.

\section{Identificação do sistema}

Neste trabalho, os algoritmos genético e de evolução diferencial são aplicados a fím de otimizar a norma $\mathrm{H}_{2}$ de um sistema torcional didático disponível no Laboratório de Controle e Otimização de Sistemas (LACOS) do Centro Integrado de Pesquisa em Controle e Automação (CIPECA) da Universidade Tecnológica Federal do Paraná (UTFPR).

A Planta Torcional ECP® - Modelo 205 é uma plataforma didática versátil para estudos de sistemas de controle. Esta planta é composta por três discos, os quais apresentam respectivos encoders, responsáveis por sua medida de posição. As unidades dos sinais de entrada e saída são dados em counts, que é a unidade utilizada nos conversores D/A e A/D. Mais informações podem ser encontradas em Parks (1999). 
O controle deste sistema é implementado utilizando o software MATLAB/Simulink ${ }^{\circledR}$ como interface de controle, permitindo assim, total versatilidade para gerar e alterar o controlador do sistema, inclusive de forma online.

Neste trabalho, a planta será implementada apenas com o Disco 1 (inferior) com duas massas de cobre localizadas a $9 \mathrm{~cm}$ do centro do disco, onde cada massa possui $0,5 \mathrm{~kg}$ e $5 \mathrm{~cm}$ de diâmetro (PARKS, 1999). Assim, o modelo em espaços de estados identificado do sistema torcional é apresentado por (5).

$$
\begin{aligned}
& {\left[\begin{array}{l}
\dot{\theta}_{c} \\
\ddot{\theta}_{c}
\end{array}\right]=\left[\begin{array}{cc}
0 & 1 \\
0 & -0,948
\end{array}\right]\left[\begin{array}{c}
\theta_{c} \\
\dot{\theta}_{c}
\end{array}\right]+\left[\begin{array}{c}
0 \\
32
\end{array}\right] u(t)} \\
& y(t)=\left[\begin{array}{ll}
49,91 & 0
\end{array}\right]\left[\begin{array}{c}
\theta_{c} \\
\dot{\theta}_{c}
\end{array}\right]
\end{aligned}
$$

Com o sistema devidamente identificado, é possível realizar seu controle em malha fechada para fins de análises de estabilidade e desempenho, o qual será tratado a seguir.

\section{Configurações experimentais}

Os algoritmos genético e de evolução diferencial são implementados pelo software MATLAB ${ }^{\circledR} \mathrm{R} 2014 \mathrm{~b}$, com um notebook Intel Core i5 7th Gen, 8GB de memória, HD de 1 TB e placa de vídeo externa NVIDIA ${ }^{\circledR}$ Geforce de 2 GB.

Para que os algoritmos possam ser implementados, faz-se necessário conhecer o modelo matemático da planta do sistema que será controlada, determinar a função objetivo a ser otimizada e, por fim, deve-se determinar os parâmetros de cada algoritmo.

Como o objetivo é encontrar um controlador que minimize a norma $\mathrm{H}_{2}$ do sistema, os algoritmos AG e ED apresentam sua função objetivo conforme a Equação (6), sendo $H(s)$ a função de transferência do controlador a ser computado.

$$
F O=\operatorname{Min}_{H(s)}\left\{\text { norma }_{2}(G(s))\right\}
$$

O sistema torcional de estudo possui ordem dois e não há necessidade de seu controlador apresentar ordem superior (OGATA; MAYA; LEONARDI, 2010). Assim, determina-se que o controlador a ser otimizado possua ordem igual à planta do sistema, ou seja, ordem dois. O modelo genérico característico deste controlador em espaço de estados pode ser ilustrado em (7).

$$
\begin{aligned}
& \dot{x}_{c}(t)=\left[\begin{array}{ll}
a & c \\
b & d
\end{array}\right] x_{c}(t)+\left[\begin{array}{l}
e \\
f
\end{array}\right] y(t) \\
& u(t)=\left[\begin{array}{ll}
g & h
\end{array}\right] x_{c}(t)+[i] y(t)
\end{aligned}
$$

Sendo: $a, b, c, d, e, f, g, h, i$ os coeficientes do controlador; $x_{c}$ os estados do controlador.

Algoritmos evolutivos utilizam progressão iterativa, ou seja, os indivíduos da população são desenvolvidos para atingirem uma adequação desejada a partir dos processos de mutação, cruzamento e seleção. Em Lacerda e Carvalho (1999) e Linden (2008) pode-se encontrar detalhadamente os processos do algoritmo genético. Em Qin, Huang e Suganthan (2009) e Price, Storn e Lampinen (2006) são apresentados os métodos para o desenvolvimento do algoritmo de evolução diferencial.

Desta forma, cada indivíduo dos algoritmos representa um controlador diferente e, seus genes representam os coeficientes deste controlador. A Figura 1 ilustra a população de indivíduos apresentada nos algoritmos.

$$
\begin{aligned}
& \begin{array}{l|l|l|l|l|l|l|l|l|l|}
\text { Controlador 1 } & a_{1} & b_{1} & c_{1} & d_{1} & e_{1} & f_{1} & g_{1} & h_{1} & i_{1} \\
\cline { 2 - 9 } \text { Controlador 2 } & a_{2} & b_{2} & c_{2} & d_{2} & e_{2} & f_{2} & g_{2} & h_{2} & i_{2} \\
\cline { 2 - 10 } \begin{array}{l}
\text { Controlador } 3 \\
\text { Controlador } 4
\end{array} & a_{3} & b_{3} & c_{3} & d_{3} & e_{3} & f_{3} & g_{3} & h_{3} & i_{3} \\
\cline { 2 - 9 } & a_{4} & b_{4} & c_{4} & d_{4} & e_{4} & f_{4} & g_{4} & h_{4} & i_{4} \\
\hline
\end{array}
\end{aligned}
$$

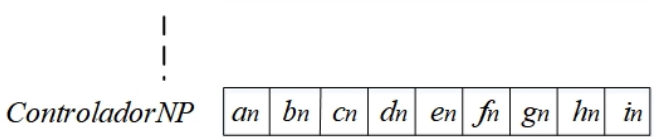

Figura 1. População dos algoritmos

Na Figura 1, an,bn,cn,dn,en, fn, gn,hn e in são os coeficientes dos controladores, como mostrados em (7).

A saída artificial inserida ao sistema é determinada a fim de especificar como deve a ponderação desejada para otimização tanto da energia interna quanto do tempo de resposta dos estados do sistema.

Assim, três testes são abordados neste trabalho, onde o primeiro especifica um tempo rápido de resposta dos estados e com uma alta energia de controle. Já o segundo teste mantém tanto o tempo de resposta, quanto a energia interna em equilíbrio e, por fim, o

\begin{tabular}{|c|c|c|c|}
\hline & Operação 1 & Operação 2 & Operação 3 \\
\hline$C_{z}$ & {$\left[\begin{array}{ll}10 & 10\end{array}\right]$} & {$\left[\begin{array}{ll}1 & 1\end{array}\right]$} & {$\left[\begin{array}{ll}1 & 1\end{array}\right]$} \\
\hline$D_{z}$ & [1] & [1] & [10] \\
\hline
\end{tabular}
terceiro teste especifica uma resposta lenta com baixa energia de controle. As ponderações relacionadas à saída artificial correspondente aos três pontos de operação estão representadas na Tabela 1 .

Tabela 1. Ponderações relacionadas à saída artificial

Para que os algoritmos AG e ED possam ser implementados, faz-se necessário conhecer seus parâmetros, os quais são apresentados nas Tabelas 2 e 3, respectivamente. Tais parâmetros foram determinados a partir de uma relação empírica probabilística.

Tabela 2. Parâmetros do algoritmo genético

\begin{tabular}{l|l}
\hline Parâmetros & 90 \\
\hline Número de indivíduos & 0,95 \\
Porcentagem de cruzamento & 0,60 \\
Taxa de cruzamento & 0,30 \\
Porcentagem de mutação & 0,10 \\
Taxa de mutação &
\end{tabular}

Tabela 3. Parâmetros do algoritmo de evolução diferencial

\begin{tabular}{l|l}
\hline Parâmetros & \\
\hline Número de indivíduos & 27
\end{tabular}




\begin{tabular}{l|l} 
Taxa de mutação & 0,45 \\
Taxa de cruzamento & 0,6 \\
\hline
\end{tabular}

Assim, com os parâmetros apresentados, os algoritmos AG e ED podem ser aplicados para a síntese de controladores. Posteriormente, é realizada a simulação do sistema em malha fechada no software MATLAB/Simulink e também fisicamente no sistema torcional a fim de validar os resultados obtidos. O Capítulo 6 apresenta os principais resultados simulados e experimentais.

\section{Resultados}

Por se tratar de meta-heurísticas, o AG e o ED não garantem o mínimo global, porém apresentam um resultado sub-ótimo dentro dos parâmetros apresentados. Assim, para obter o controlador otimizado, foram realizados dez ciclos do algoritmo, onde será considerado o melhor controlador para cada ponto de operação dentre os dez ciclos realizados, ou seja, o controlador que resulta na menor norma do sistema.

Tanto os controladores sintetizados pelo $\mathrm{AG}$ quanto os controladores sintetizados pelo ED não acionaram o atuador do sistema torcional. Notou-se também, que os polos do sistema em malha fechada nestas condições eram muito negativos o que caracteriza uma busca rápida dos estados, porém, conflitante com a baixa energia aplicada.

A solução encontrada foi a atribuição de penalidades na função objetivo dos algoritmos, a fim de ponderá-los. A Equação (8) mostra a nova função objetivo aplicada.

$$
F O=\operatorname{Min}_{H(s)}\left\{\text { norma } H_{2}(G(s))-\operatorname{Min}\left|\lambda_{i}\right|+\operatorname{Max}\left|\lambda_{i}\right|\right\},
$$

sendo: $\operatorname{Min}\left|\lambda_{i}\right|$ o polo de menor módulo; $\operatorname{Max}\left|\lambda_{i}\right| \mathrm{o}$ polo de maior módulo do sistema em malha fechada.

Com a nova função objetivo devidamente apresentada ao algoritmo genético e ao algoritmo de evolução diferencial, torna-se possível implementá-los a fim de obter controladores otimizados e que sejam passíveis de realizar o controle em malha fechada do sistema físico estudado.

\subsection{Algoritmo genético}

Para o primeiro ponto de operação estipulado, o controlador que obteve o melhor valor de função objetivo é apresentado em (9), sendo seu valor igual a 42,9246.

$$
\begin{aligned}
& \dot{x}_{c}=\left[\begin{array}{cc}
-59,4138 & 4,0125 \\
-12,7890 & -38,2653
\end{array}\right] x_{c}+\left[\begin{array}{c}
5,2727 \\
3,0492
\end{array}\right] y \\
& u=\left[\begin{array}{ll}
4,4320 & 7,8228
\end{array}\right] x_{c}+[-0,9399] y
\end{aligned}
$$

A fim de validar tais controladores, aplicou-se à entrada um sinal degrau com amplitude de 1000 counts durante 5 segundos. Torna-se interessante também, analisar a energia de controle deste sistema, já que este é um dos parâmetros de otimização.
Assim, o sinal de saída deste sistema à referência degrau, bem como o sinal de sua energia e controle em malha fechada é apresentado pela Figura 2.
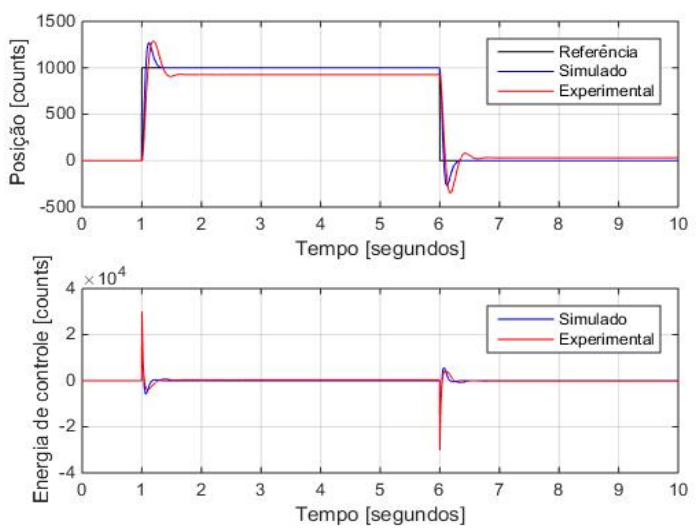

Figura 2. Resposta ao degrau e energia de controle Operação $1 \mathrm{AG}$

Nota-se um erro de 7,3\% de seu valor em regime, porém, com resultados ainda satisfatórios.

Já para o segundo ponto de operação, a menor função objetivo é igual a 8,6465, sendo seu controlador correspondente expresso por (10).

$$
\begin{aligned}
& \dot{x}_{c}=\left[\begin{array}{cc}
-19,0485 & 2,3525 \\
-13,2716 & -51,4745
\end{array}\right] x_{c}+\left[\begin{array}{l}
6,9437 \\
0,9005
\end{array}\right] y \\
& u=\left[\begin{array}{ll}
4,6738 & 12,6241] x_{c}+[-1,1194] y
\end{array}\right.
\end{aligned}
$$

A resposta de sua saída à entrada degrau já estipulada é apresenta na Figura 3 juntamente com a energia do sistema em malha fechada.

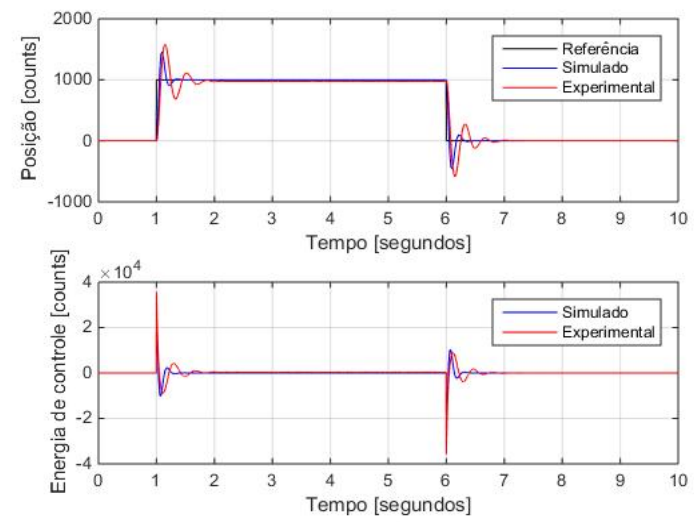

Figura 3. Resposta ao degrau e energia de controle Operação 2 AG

Pode-se aferir que o controlador foi eficiente e apresentou erro em regime ainda menor do que o controlador apresentado no ponto de operação anterior, sendo em torno de $2,5 \%$ de seu valor em regime.

Por fim, o terceiro e último ponto de operação apresenta o controlador mais otimizado conforme (11) , cuja função objetivo é igual a 27,9722.

$$
\begin{aligned}
& \dot{x}_{c}=\left[\begin{array}{ll}
-28,3142 & -4,5028 \\
-5,8870 & -38,3134
\end{array}\right] x_{c}+\left[\begin{array}{l}
-2,2705 \\
-6,6171
\end{array}\right] y
\end{aligned}
$$

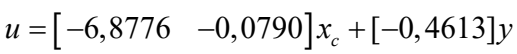

A resposta em malha fechada ao se aplicar este controlador é apresentada na Figura 4. 

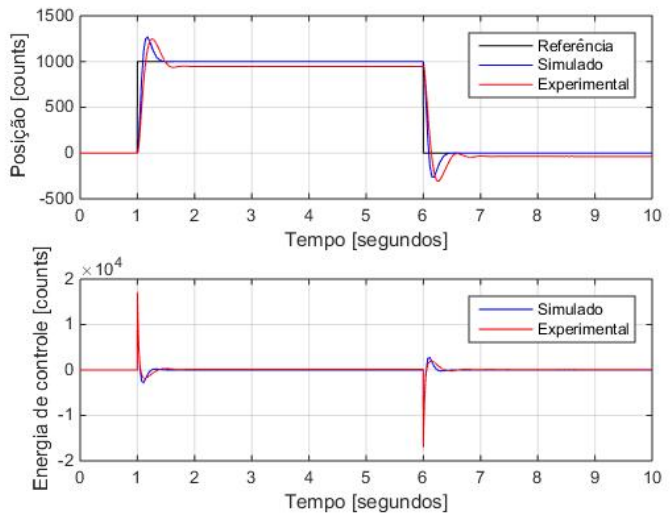

Figura 4. Resposta ao degrau e energia de controle Operação 3 AG

Tal controlador apresenta erro em regime maior, sendo aproximadamente $5,4 \%$ de seu valor. A seguir são apresentados os controladores e suas respostas obtidas pelo algoritmo de evolução diferencial.

\subsection{Algoritmo de evolução diferencial}

Para o primeiro ponto de operação estipulado, o controlador que obteve o melhor valor de função objetivo é apresentado em (12), sendo seu valor igual a 39,1924 .

$$
\begin{aligned}
& \dot{x}_{c}=\left[\begin{array}{rr}
-34,7649 & -0,3702 \\
-73,2970 & -11,9914
\end{array}\right] x_{c}+\left[\begin{array}{l}
-1,4291 \\
-0,2840
\end{array}\right] y \\
& u=\left[\begin{array}{ll}
-6,5510 & -0,1389
\end{array}\right] x_{c}+[-0,3501] y
\end{aligned}
$$

Assim, o sinal de saída deste sistema e sua energia e controle em malha fechada é apresentado pela Figura 5 .
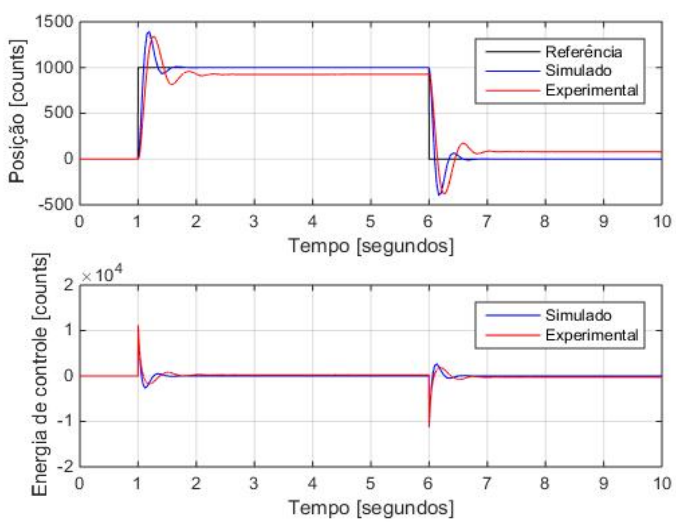

Figura 5. Resposta ao degrau e energia de controle Operação 1 ED

Nota-se apenas um erro de aproximadamente $7,4 \%$ de seu valor em regime, porém, com resultados ainda satisfatórios, pois a referência foi seguida.

Já para o segundo ponto de operação, a menor função objetivo é igual a 5,1162, sendo seu controlador correspondente expresso por (13).

$$
\begin{aligned}
\dot{x}_{c} & =\left[\begin{array}{cc}
-30,2376 & 7,9814 \\
-34,2891 & -39,2987
\end{array}\right] x_{c}+\left[\begin{array}{c}
-1,0005 \\
3,8490
\end{array}\right] y \\
u & =\left[\begin{array}{ll}
5,4532 & 3,1185
\end{array}\right] x_{c}+[-0,3906] y
\end{aligned}
$$

A resposta de sua saída à entrada degrau já estipulada é apresenta na Figura 6 juntamente com a energia do sistema em malha fechada.
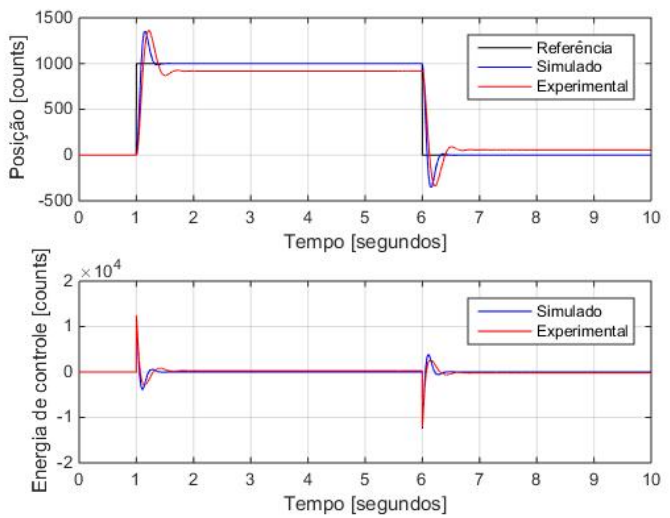

Figura 6. Resposta ao degrau e energia de controle Operação 2 ED

Pode-se aferir que o controlador foi eficiente e apresentou erro em regime ainda menor do que o controlador apresentado no ponto de operação anterior, sendo em torno de $8,2 \%$ de seu valor em regime.

Por fim, o terceiro e último ponto de operação apresenta o controlador mais otimizado conforme (14) , cuja função objetivo é igual a 25,6131.

$$
\begin{aligned}
& \dot{x}_{c}=\left[\begin{array}{cc}
-18,5071 & -27,6530 \\
12,5730 & -29,4228
\end{array}\right] x_{c}+\left[\begin{array}{l}
0,8634 \\
2,1820
\end{array}\right] y \\
& u=\left[\begin{array}{ll}
-2,2125 & 0,3104] x_{c}+[-0,1884] y
\end{array}\right.
\end{aligned}
$$

A resposta em malha fechada ao se aplicar este controlador é apresentada na Figura 7.
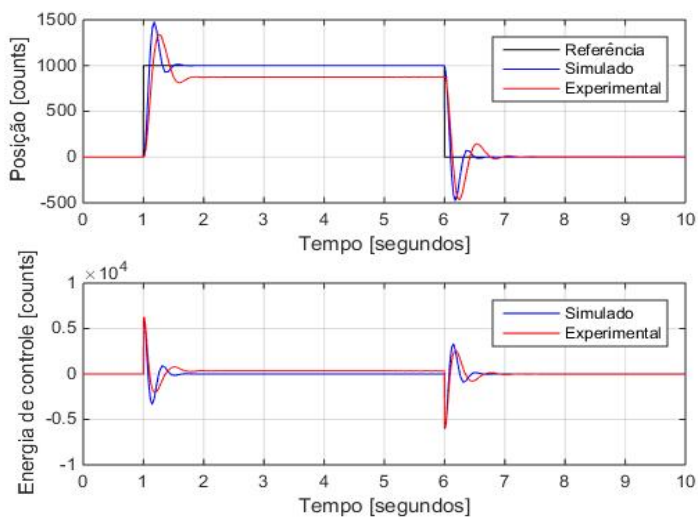

Figura 7. Resposta ao degrau e energia de controle Operação 3 ED

Conforme a Figura 7, tal controlador apresenta erro em regime maior do que os controladores apresentados nos pontos de operação anteriores, sendo aproximadamente $12,5 \%$ de seu valor.

\section{Conclusão}

Este trabalho propôs a utilização de dois métodos de otimização aplicados a sistemas de controle, sendo eles o algoritmo genético e o algoritmo de evolução diferencial. Ambos algoritmos objetivaram o cálculo 
de controladores que resultassem na minimização da norma $\mathrm{H}_{2}$ de sistemas de controle.

Apesar das penalidades inseridas na função objetivo, os algoritmos genéticos e de evolução diferencial se mostram eficientes alternativas às soluções algébricas como LMIs e de Riccati, pois as alterações necessárias foram realizadas de forma simples. Se a mesma restrição fosse aplicada às soluções por LMIs ou Riccati, diversas equações algébricas deveriam ser refeitas, o que aumentaria a complexidade de sua solução, possivelmente gerando técnicas conservadoras.

Alguns pontos de operação foram analisados para diferentes configurações relacionando a resposta dos estados e a energia de controle. Com os resultados obtidos, notou-se que ao considerar a mesma ponderação obteve-se o menor valor da função objetivo e também respostas com menor erro em regime. Assim, torna-se interessante aplicar as matrizes $C_{z}$ e $D_{z}$ de forma igualitária na otimização de controladores a partir de algoritmos evolutivos, conforme o disposto no ponto de operação descrito.

O tempo de convergência entre ambos algoritmos são muito discrepantes, sendo que o algoritmo genético leva em média 18,7 minutos para sua convergência, enquanto o algoritmo de evolução diferencial demora em média 6,2 minutos.

O algoritmo de evolução diferencial também apresenta menores valores médios de sua função objetivo, em comparação com o algoritmo genético, o que o caracteriza como uma melhor ferramenta de otimização.

Portanto, pode-se concluir que os algoritmos genéticos e de evolução diferencial são eficientes ferramentas aplicadas na otimização de controladores, apesar das restrições necessárias. Cabe ao usuário conhecer as aplicações do sistema, a fim de definir qual dos dois algoritmos deve ser utilizado.

\section{Referências Bibliográficas}

Andrade, L. H. S. de, Agulhari, C. M. (2016). Robust mixed $\mathrm{H}_{2} / \mathrm{H}_{\infty}$ control of an nonlinear multivariable system. 12th IEEE/IAS International Conference on Industry Application, Curitiba - Brasil.

Boers, Y., Weiland, S., Damen, A. A. H. (1997). Optimal expected $\mathrm{H}_{2}$ performance controllers. European Control Conference, Brussels - Belgium.

Boyd, S., Ghaovi, L. E., Feron, E., Balakrishnan, V. (1994). Linear matrix inequalities in system and control theory. Society for industrial and applied mathematics, Philadelphia.

Chun, S., Choi, G., Kim, T. (2017). Design of mixed static/dynamic output feedback controller using multi-objective distribute particle swarm optimization. 11th Asian Control Conference, Gold Coast Convention Centre - Australia.

Das, S., Sugaratnam, P. N. (2011). Differential evolution: a survey of the state-of-the-art. IEEE transactions on evolutionary computation, vol. 15, n. 1, pp. 4-31.

Farias, D. P. de, Geromel, J. C., Val, J. B. R. do, Costa, O. L. V. (2000). Output feedback control of Markov jump linear systems in continuous-time. IEEE transactions on Automatic Control, vol. 45, pp. 944-949.
Ghaoui, L. E., Niculescu, S.-L. (2000). Advances in linear matrix inequality methods in control. Society for industrial and applied mathematics, Philadelphia.

Gomez, M. A., Egorov, A. V., Mondié, S., Michiels, W. (2015). Optimization of the $\mathrm{H}_{2}$ norm for single delay systems, with application to control design and model approximation. Journal of LATEX class files, vol. 14, n. 8, pp. 1-8.

Gonçalves, E. N., Palhares, R. M., Takahashi, R. H. C. (2004). Multi-objective optimization applied to robust $\mathrm{H}_{2} / \mathrm{H}_{\infty}$ state-feedback control synthesis. American Control Conference, Boston - EUA.

Kao, Y., Zahara, E. (2007). A hybrid genetic algorithm and particle swarm optimization for multimodal functions. Applied Soft Computing, vol. 8, pp. 849-857.

Lacerda, E. G. M. de, Carvalho, A. C. P. F. L. de. (1999). Introdução aos algoritmos genéticos. Editora da Universidade da UFRGS - ABRH, Porto Alegre - Brasil.

Leibfritz, F. (2001). An LMI-based algorithm for designing subotimal static $\mathrm{H}_{2} / \mathrm{H}_{\infty}$ output feedback controllers. SIAM J. Control Optim, vol. 39, no. 6, pp. 1711-1735.

Linden, R. Algoritmos genéticos. (2008). 1 ed. Ei Edições Inteligentes, São Paulo - Brasil.

Ogata, K., Maya, P. A., Leonardi, F. (2010). Engenharia de controle moderno. 3 ed. Prentice Hall.

Parks, T. R. (1999). Manual for Model 205/205a: Torcional control system. Educational Control Products. Bell Canyon.

Price, K., Storn, R. M., Lampinen, J. A. (2006). Differential evolution: a practical approach to global optimization. Springer Science \& Business Media.

Qin, A. K., Huang, V. L., Sugantham, P. N. (2009). Differential evolution algorithm with strategy adaptation foe global numerical optimization. IEEE transactions on evolutionary computation, vol. 13, n. 2, pp. 398-417.

Sato, M., Ebihara, Y., Peaucelle, D. (2010). Gain-scheduled state-feedback controllers using inexactly measured scheduling parameter: $H_{2}$ and $H_{\infty}$ problems. American Control Conference, Baltimore, EUA.

Trofino, A., Coutinho, D. F., Barbosa, K. A. (2005). Improved $\mathrm{H}_{2}$ and $H_{\infty}$ conditions for robust analysis and control synthesis of linear systems. Revista Controle e Automação, vol. 16, n. 4, pp. 427-434.

Wu, L., Wang, Y., Shou, S., Yuan, X. (2013). Design of mixed $\mathrm{H}_{2} / \mathrm{H}_{\infty}$ optimal control systems using multi-objective differential evolution algorithm. J Control Theory Appl, vol. 11, n. 3, pp. 521-528.

Xie, W., Cheng, Y. (2010). Simultaneous $H_{2}$ design of structural and controller parameters with state-feedback. 8th World Congress on Intelligent Control and Automation, Jian - China.

Yu, J.-T. (2004). A new static output feedback approach to the suboptimal mixed $\mathrm{H}_{2} / \mathrm{H}_{\infty}$ problem. International Journal of Robust and Nonlinear Control, vol. 14, pp. 1023-1034.

Zhou, K., Doyle, J. C., Glover, K. (1996). Robust and optimal control. Prentice Hall, Englewood Cliffs, New Jersey. 